\title{
Determination of Internal Loss and Quasi-Fermi Level Separation From the Amplified Spontaneous Emission Spectrum of Fabry-Pérot Semiconductor Lasers
}

\author{
W. H. Guo, D. Byrne, Q. Y. Lu, R. Phelan, J. F. Donegan, and B. Corbett
}

\begin{abstract}
The net modal gain and the un-amplified spontaneous emission (ASE) coupled into the laser waveguide mode are extracted from the ASE spectrum of Fabry-Pérot semiconductor lasers by the Fourier transform method with a deconvolution process. Highly accurate quasi-Fermi level separation and internal loss are then derived by a minimum search process from the relationship between the spontaneous emission and gain.
\end{abstract}

Index Terms-Amplified spontaneous emission (ASE), Fabry-Pérot (FP) semiconductor laser, Fourier transform (FT) method, internal loss, quasi-Fermi level separation.

\section{INTRODUCTION}

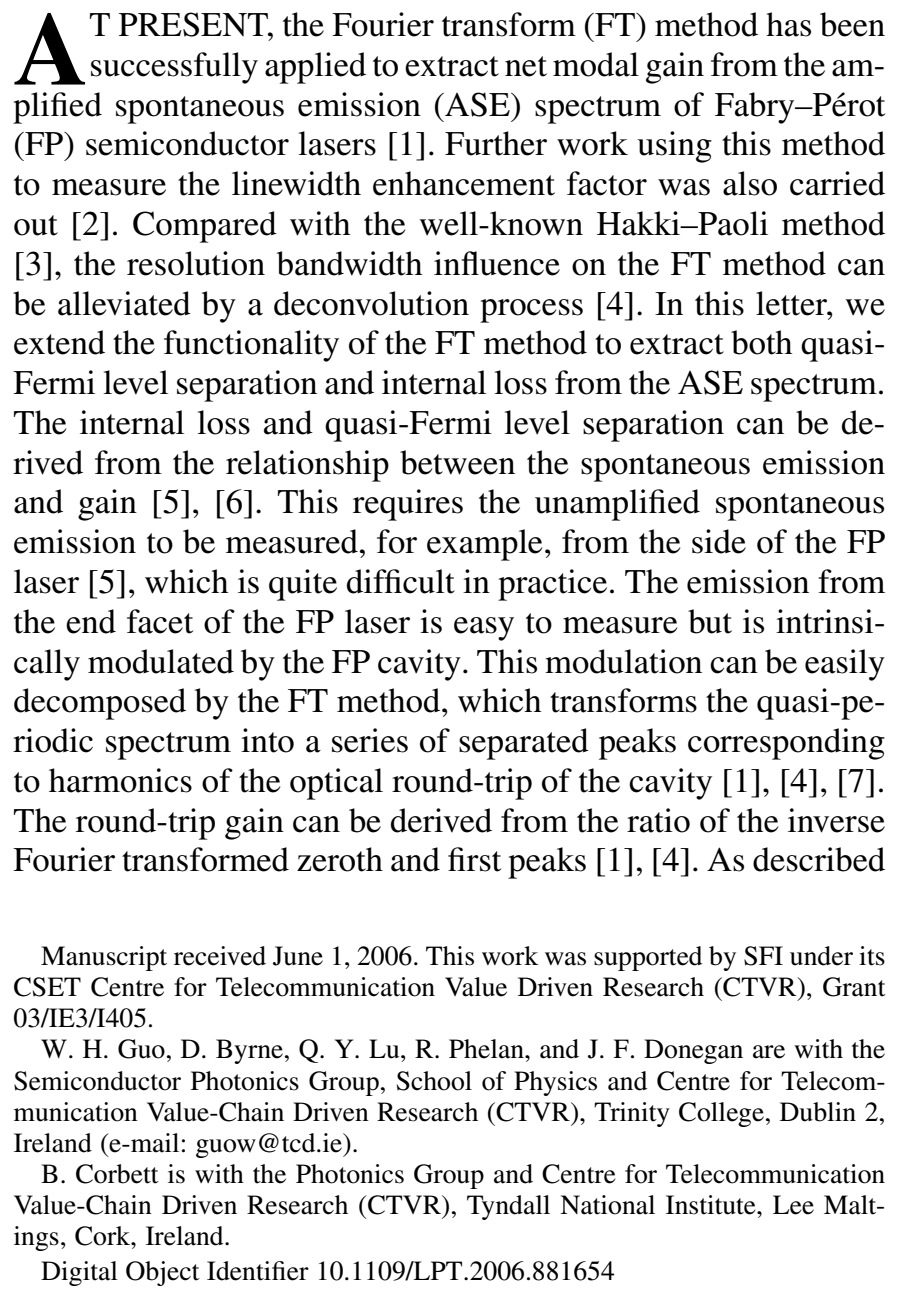

below, the zeroth harmonic also contains the spontaneous emission information [6] which, however, is omitted in the standard FT method. In this letter, we use a minimum search process to extract the internal loss and quasi-Fermi level separation from the spontaneous emission and the net modal gain spectrum, both of which are obtained from the FT method. The gain extraction always needs a high resolution bandwidth, otherwise the underestimation of the gain will have a negative influence on the accuracy of the extracted internal loss and quasi-Fermi level separation. We also show that by using a deconvolution process, the gain underestimation can be reduced [4] so that the requirement for high resolution bandwidth can be eased.

\section{THEORY}

The ASE spectrum of FP semiconductor lasers can be described by [8], [9]

$$
I=\frac{I_{\mathrm{sp}}(1-R)(1+b)}{1+b^{2}-2 b \cos \left(2 n_{\mathrm{eff}} k L\right)}
$$

where $b=\exp (g L) R$ is the round-trip gain, $g$ is the net modal gain; $L$ is the cavity length; $R$ is the power reflectivity of the end-facet; $k=2 \pi / \lambda$ is the wavenumber in vacuum

$$
I_{\mathrm{sp}}=\frac{S[\exp (g L)-1]}{g}
$$

is the single pass ASE, $S$ is related to the spontaneous emission rate $r_{\mathrm{sp}}$ by $S=A \beta r_{\mathrm{sp}} h c / \lambda, A$ is the transverse area of the quantum wells (QWs), $\beta$ is the ratio of the spontaneous emission coupled into the waveguide mode. The FT of the modulated ASE spectrum (1) is composed of a series of separated peaks, which correspond to harmonics of the optical round-trip of the FP cavity. Selecting the zeroth and first harmonics and taking their inverse FT, the following spectra can be obtained:

$$
\begin{aligned}
& I_{0}=\left|\int_{-n_{\mathrm{eff}} L}^{n_{\mathrm{eff}} L} \widetilde{I}(z) \exp (i k z) d z\right|=\frac{I_{\mathrm{sp}}(1-R)}{1-b} \\
& I_{1}=\left|\int_{n_{\mathrm{eff}} L}^{3 n_{\mathrm{eff}} L} \widetilde{I}(z) \exp (i k z) d z\right|=b I_{0}
\end{aligned}
$$


where $\sim$ above indicates a Fourier transformed quantity. The round-trip gain can consequently be calculated as $b=I_{1} / I_{0}[1]$. This is the fundamental aspect of the FT method for gain extraction. The net modal gain can be calculated as $g=\log (b / R) / L$. Combining (2) and (3) the spontaneous emission coupled into the laser waveguide mode, $S$ in (2), is also obtained

$$
S=\frac{I_{0}(1-b) g}{(1-R)[\exp (g L)-1]} .
$$

The relationship between the spontaneous emission rate and the material gain is [5]

$$
g_{m}=\frac{\lambda^{4}}{8 \pi c n^{2}}\left[1-\exp \left(\frac{\frac{h c}{\lambda}-\Delta F}{k_{B} T}\right)\right] r_{\mathrm{sp}}
$$

where $\Delta F$ is the quasi-Fermi level separation. The material gain is related to the net modal gain through $g=\Gamma g_{m}-\alpha_{\text {in }}, \alpha_{\text {in }}$ is the internal loss. Now combining the above relations we can obtain

$$
\begin{aligned}
g(\lambda) & =C \lambda^{5} S\left[1-\exp \left(\frac{\frac{h c}{\lambda}-\Delta F}{k_{B} T}\right)\right]-\alpha_{\text {in }} \\
& \equiv C q(\lambda, \Delta F)-\alpha_{\text {in }}
\end{aligned}
$$

where $C=\Gamma /\left(8 \pi c^{2} n^{2} h \beta A\right)$. From (7), it is seen that the net modal gain $g(\lambda)$ is a linear function of $q(\lambda, x)$ as $x=\Delta F$. This point can be used to extract the quasi-Fermi level separation $\Delta F$. The extraction process is as follows: scanning $x$ within a range in which $\Delta F$ is estimated; for each value of $x$, linearly fitting $[q(\lambda, x), g(\lambda)]$ and monitoring the norm of the residuals; determining $\Delta F$ from the minimum of the norm; determining $\alpha_{\text {in }}$ from the intercept of the linear fit with $x=\Delta F$. The experimentally measured spectrum is the laser ASE spectrum convoluted by the response function of the measurement system. The gain will be underestimated if the resolution bandwidth used is not high enough. However, with a deconvolution process [4], even a moderate resolution bandwidth can produce a satisfactory result. The deconvolution process yields modified equations of (3) and (4)

$$
\begin{aligned}
I_{0} & =\int_{-n_{\text {eff }} L}^{n_{\text {eff }} L} \frac{\widetilde{I}(z)}{2 \pi \widetilde{f}^{*}\left(\frac{k^{2}}{2 \pi} z\right)} \exp (i k z) d z \\
I_{1} & =\int_{n_{\mathrm{eff}} L}^{3 n_{\mathrm{eff}} L} \frac{\widetilde{I}(z)}{2 \pi \widetilde{f}^{*}\left(\frac{k^{2}}{2 \pi} z\right)} \exp (i k z) d z
\end{aligned}
$$

where $*$ means complex conjugate, $f(x)$ is the response function of the measurement system which can be obtained by measuring a single wavelength source with a linewidth much narrower than the designated resolution bandwidth.

\section{Simulation AND MeASUREMENT}

To check the above scheme, we performed some numerical simulations first. An FP semiconductor laser with the active region composed of six strain-compensated QWs was simulated.

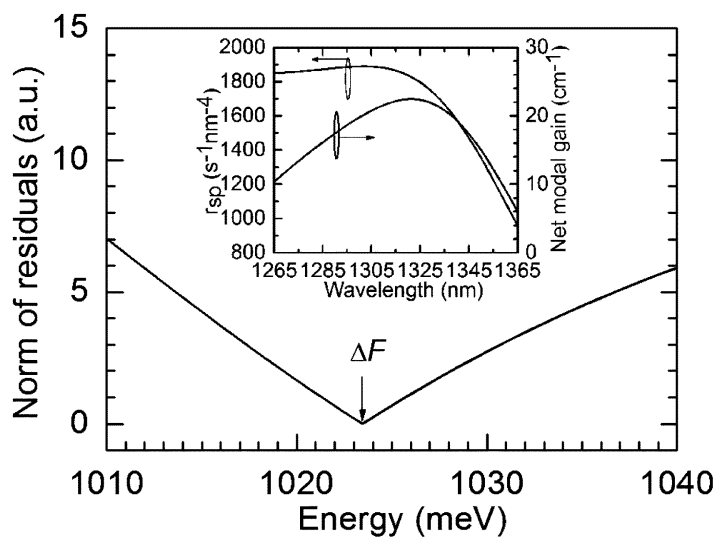

Fig. 1. Norm of residuals from linear fit versus $x$. Inset: spontaneous emission and net modal gain spectrum used in the simulation.

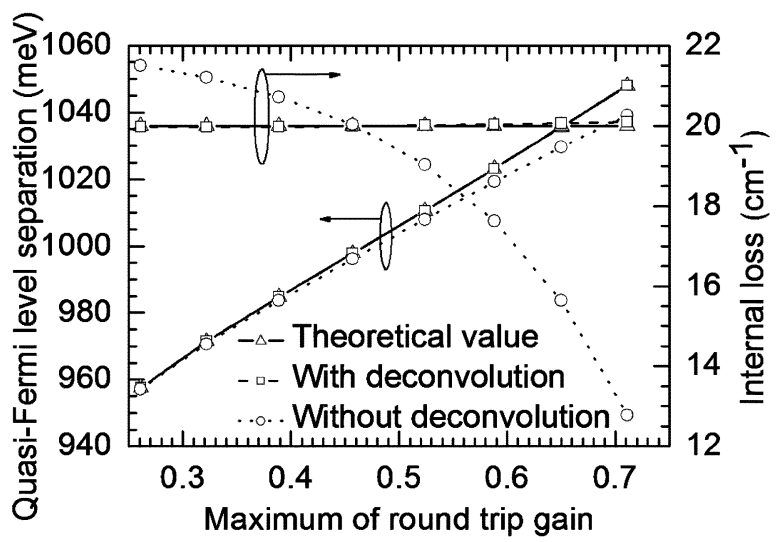

Fig. 2. Extracted quasi-Fermi separations and internal loss versus maximal round-trip gain with and without the deconvolution process. The theoretical values are also shown for comparison.

The gain and the spontaneous emission spectrum were calculated taking account of valence-band mixing and many-body effects [5]. The ASE spectrum was produced through (1) with the cavity length $300 \mu \mathrm{m}$ and a group index 3.61. The simulated ASE spectrum was analyzed by the FT method to extract the net modal gain and the spontaneous emission spectrum. Through the minimum search process introduced above we determined the quasi-Fermi level separation and the internal loss. In the simulation, we first set the parameters $\Delta F=1023.18 \mathrm{meV}$ and $\alpha_{\text {in }}=20 \mathrm{~cm}^{-1}$. The extracted values were $1023.43 \mathrm{meV}$ and $20.02 \mathrm{~cm}^{-1}$, so very high accuracy was obtained. The norm of the residuals is plotted in Fig. 1, which shows the minimum indicating the quasi-Fermi level separation $\Delta F$. The inset shows the spontaneous emission and the net modal gain spectrum used in the simulation, which cover a range of $100 \mathrm{~nm}$ around the gain peak. To show the resolution bandwidth influence, we then convoluted the simulated ASE spectrum with a triangular response function [9] which has a full-width at half-maximum (FWHM) of $0.1 \mathrm{~nm}$. The carrier density in the QWs was varied and this produced different maximal round-trip gain for the FP cavity. The quasi-Fermi level separation and the internal loss were extracted with and without the deconvolution process and the results are shown in Fig. 2 versus the maximal round-trip gain. It is seen that without the deconvolution process, the quasi-Fermi 


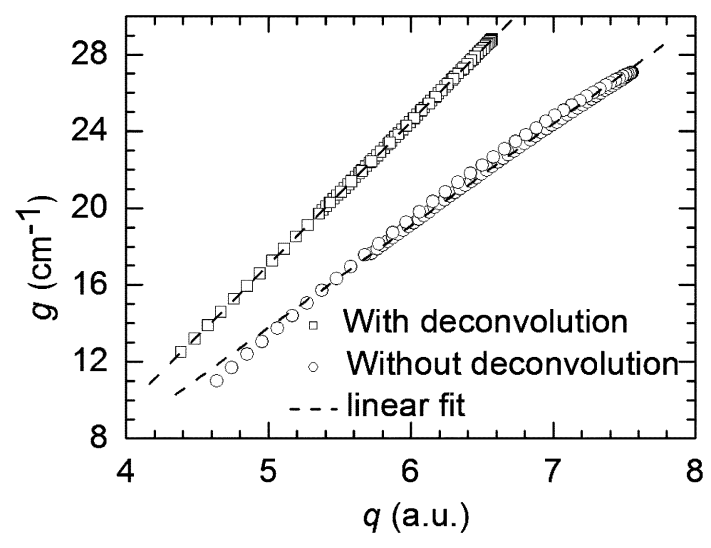

Fig. 3. $[q(\lambda, \Delta F), g(\lambda)]$ curve obtained with and without the deconvolution process along with the linear fit.

level separation is always underestimated and the underestimation is severe for high round-trip gain situations. The internal loss is overestimated in the low round-trip gain case and underestimated in the high gain case. Overall the accuracy is better for low gain situations. However, with the deconvolution process, the extracted parameters are always obtained with high accuracy, which means that the requirement for high resolution bandwidth can be greatly eased by this process. In Fig. 3, we plot the $[q(\lambda, \Delta F), g(\lambda)]$ curve along with the linear fit. The maximal round-trip gain in this case was 0.711 . It is seen that without the deconvolution process, the final curve is nonlinear indicating that the resolution used is not high enough.

In the following, we measured a practical FP ridge waveguide laser operating around $1300 \mathrm{~nm}$. A single-mode fiber with an antireflection-coated lens on its head was used to couple the ASE power into an optical spectrum analyzer (OSA) (Agilent 86140B). The laser length was measured to be $300 \mu \mathrm{m}$. The laser is mounted on a heat sink and was held at a constant temperature of $20^{\circ} \mathrm{C}$ using a thermoelectric cooling unit. We do not use a polarizer here because the QWs used in the laser are highly compressively strained and the TM emission can be neglected. The threshold current of the laser is about $12 \mathrm{~mA}$. The nominal resolution bandwidth of the OSA was set to be $0.2 \mathrm{~nm}$. The true resolution bandwidth determined from the FWHM of the OSA response function around $1300 \mathrm{~nm}$ is $0.176 \mathrm{~nm}$ which is about $23 \%$ of the longitudinal mode spacing. The ASE spectrum covering a range of about $100 \mathrm{~nm}$ around the gain peak was recorded and averaged over 20 times to improve the signal-tonoise ratio. The Hanning window function was multiplied to the measured spectrum to soften the intensity variation from the center to the edge, which can reduce the derived gain fluctuations at the edge of the measurement window [4]. Fig. 4 shows the internal loss and the quasi-Fermi-level separation extracted with and without the deconvolution process. It is seen that with the deconvolution process the internal loss extracted is more reliable. The value slightly increases with increasing current and almost saturates as the current comes close to threshold. As the simulation shows, insufficient resolution bandwidth results in overestimation of the internal loss in the low gain case and underestimation in the high gain case. The quasi-Fermi level separation, however, is always underestimated.

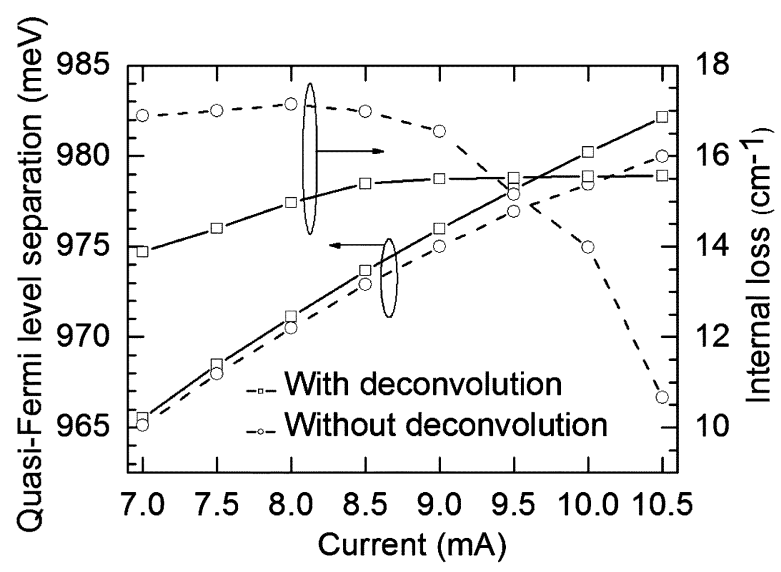

Fig. 4. Measured quasi-Fermi level separations and internal loss versus current with and without the deconvolution process.

\section{CONCLUSION}

We propose a minimum search process to extract the internal loss and quasi-Fermi-level separation from the ASE spectrum of FP semiconductor lasers. The FT method was used to extract the net modal gain and the unamplified spontaneous emission coupled into the laser waveguide mode. By using a deconvolution process the net modal gain can be obtained almost free from the influence of the resolution bandwidth, which yields more accurate internal loss and quasi-Fermi level separations.

\section{ACKNOWLEDGMENT}

The authors would like to thank Eblana Photonics for the supply of the FP laser used in this study.

\section{REFERENCES}

[1] D. Hofstetter and J. Faist, "Measurement of semiconductor laser gain and dispersion curves utilizing Fourier transform of the emission spectra," IEEE Photon. Technol. Lett., vol. 11, no. 11, pp. 1372-1374, Nov. 1999.

[2] K. G. Gan and J. E. Bowers, "Measurement of gain, group index, group velocity dispersion, and linewidth enhancement of an InGaN multiple quantum-well laser diode," IEEE Photon. Technol. Lett., vol. 16, no. 5, pp. 1256-1258, May 2004.

[3] B. W. Hakki and T. L. Paoli, "Gain spectra in GaAs double-heterostructure injection lasers,” J. Appl. Phys., vol. 46, pp. 1299-1305, 1975.

[4] W. H. Guo, Y. Z. Huang, C. L. Han, and L. J. Yu, "Measurement of gain for Fabry-Pérot semiconductor lasers by the Fourier transform method with a deconvolution process," IEEE J. Quantum Electron., vol. 39, no. 6, pp. 716-721, Jun. 2003.

[5] J. Minch, S. H. Park, T. Keating, and S. L. Chuang, "Theory and experiment of $\mathrm{In}_{1-x} \mathrm{Ga}_{x} \mathrm{As}_{y} \mathrm{P}_{1-y}$ and $\mathrm{In}_{1-x-y} \mathrm{Ga}_{x} \mathrm{Al}_{y}$ As long-wavelength strained quantum-well lasers," IEEE J. Quantum Electron., vol. 35, no. 5, pp. 771-782, May 1999.

[6] L. Fu, L. Wu, and H. Schweizer, "The extension of gain spectra and accurate determination of the quasi-Fermi-level separation from measured amplified spontaneous emission spectra," Appl. Phys. Lett., vol. 75, pp. 3069-3071, 1999.

[7] P. Lambkin, C. Percival, and B. Corbett, "Reflectivity measurements of intracavity defects in laser diodes," IEEE J. Quantum Electron., vol. 40, no. 1, pp. 10-17, Jan. 2004.

[8] C. S. Chang, S. L. Chuang, J. R. Minch, W. W. Fang, Y. K. Chen, and T. Tanbun-Ek, "Amplified spontaneous emission spectroscopy in strained quantum-well lasers," IEEE J. Sel. Toptics Quantum Electron., vol. 1, no. 4, pp. 1100-1107, Dec. 1995.

[9] W. H. Guo, Q. Y. Lu, Y. Z. Huang, and L. J. Yu, "Fourier series expansion method for gain measurement from amplified spontaneous emission spectra of Fabry-Pérot semiconductor lasers," IEEE J. Quantum Electron. Lett., vol. 40, no. 2, pp. 123-129, Feb. 2004. 